Paediatr. Paedolog. 2021 · 56:24-29 https://doi.org/10.1007/s00608-020-00860-8 Angenommen: 21. Dezember 2020 Online publiziert: 21. Januar 2021

๑) Springer-Verlag GmbH Austria, ein Teil von Springer Nature 2021

Josef Emhofer - Barbara Emhofer - Evelyn Lechner

'kokon - Reha für junge Menschen, Rohrbach, Österreich

\title{
kokon - Rehabilitation für junge Menschen in Rohrbach-Berg
}

dings keine Mutter- oder Vater-KindKuren; im Mittelpunkt stehen immer die chronisch erkrankten Kinder und Jugendlichen in einer ganzheitlichen oder systemischen Sichtweise. Das Rehabilitationsprogramm ist komplett auf die Bedürfnisse der Kinder und Jugendlichen ausgerichtet. Ein wesentlicher Ansatz des Reha-Konzepts ist, dass die Behandlung und Betreuung durch Spezialisten stattfindet, die viel Expertise und Feingefühl für die Arbeit mit Kindern und Jugendlichen mitbringen und sie individuell auf dem Weg zu ihren Zielen begleiten.

Von allen vergleichbaren Zentren in Österreich bieten wir mit kokon das breiteste Spektrum an: Neben dem kompletten Bereich der mobilisierenden Reha (36 Betten, 3-4 Wochen Aufenthaltsdauer) liegen unsere Schwerpunkte in der Betreuung sämtlicher kardiologischer und pulmologischer Krankheitsbilder (17 Betten, 3 Wochen) sowie in der MentalHealth-Rehabilitation (24 Betten, 5 Wo- chen). Als Schwerpunkte haben wir uns für das Jahr 2020 frühkindliche Essstörungen inklusive Sondenentwöhnung, Skoliose, zystische Fibrose sowie Mental Health gesetzt und für 2021 unter anderem Reha bei nephrologischen Erkrankungen und Mobilisation nach Verletzungen, Verbrennungen und Operationen.

\section{Unterschiedlichste Rehabilita- tionsschwerpunkte}

So umfangreich wie die Schwerpunkte sind auch die ärztlichen und therapeutischen Behandlungsteams unter der Leitung von PD Dr. Evelyn Lechner mit ihren Spezialgebieten Kinderkardiologie, Neonatologie und pädiatrische Intensivmedizin. Dazu kommen weitere Kinder- und Jugendfachärzte mit den Sonderfächern Neuropädiatrie, Pulmologie und Allergologie, Pädiatrische Kardiologie, Nephrologie sowie Ärzte für Allgemeinmedizin. Den MentalVerfügung. Dazu kommen 67 Betten fur Begleitpersonen. kokon erbringt aller-

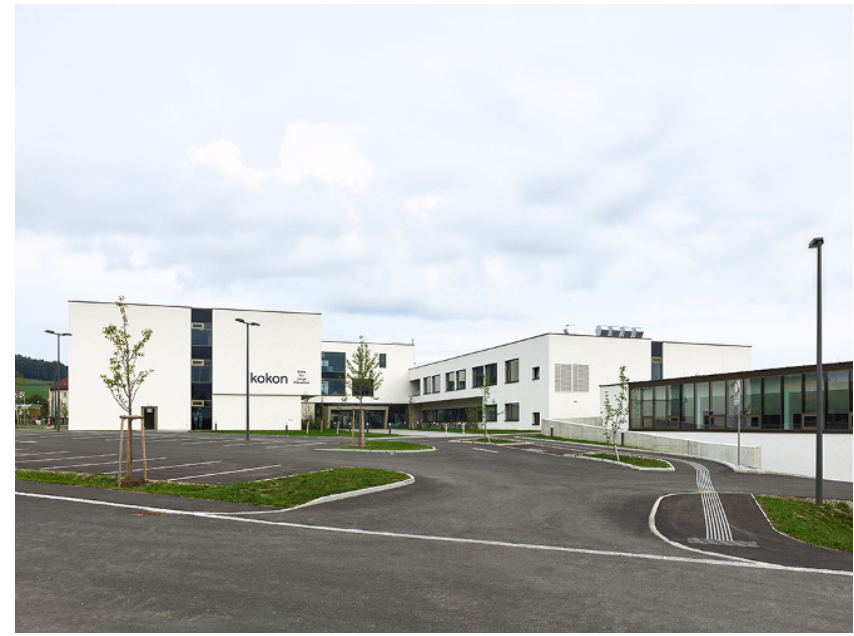

Abb. $1 \Delta$ kokon - Reha für junge Menschen in Rohrbach-Berg in Oberösterreich (@ kokon - Reha für junge Menschen)

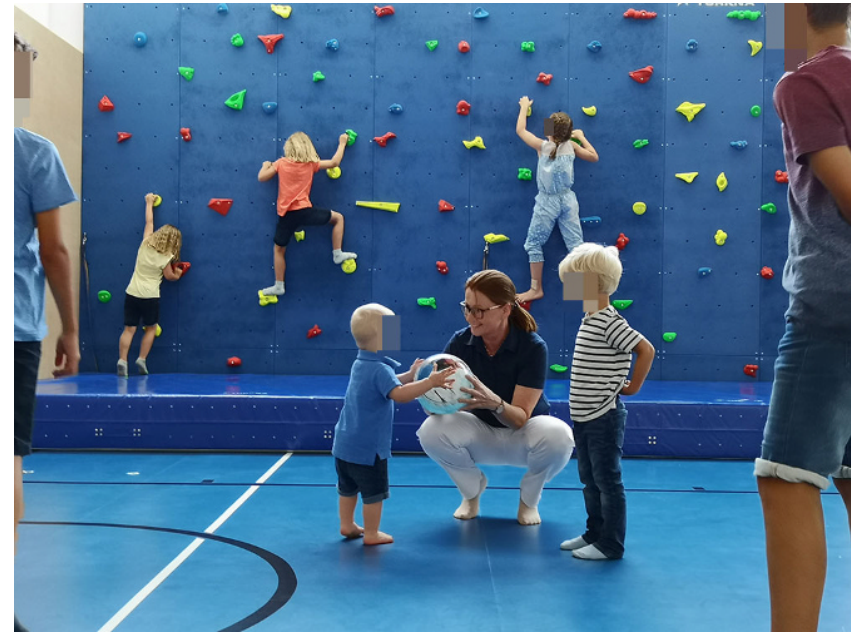

Abb. 2 × Turnsaal im kokon (@ kokon - Reha für junge Menschen) 


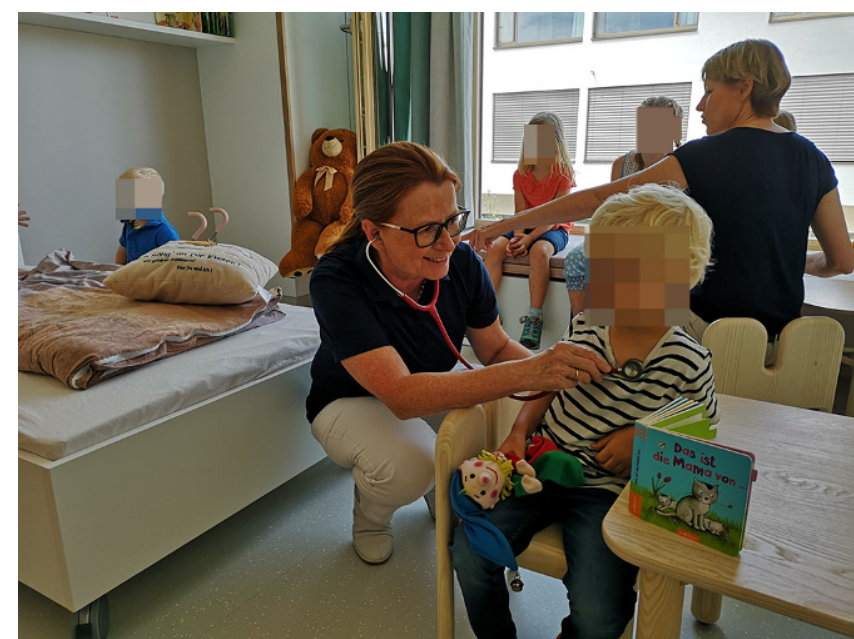

Abb. $3 \Delta$ Zimmer im kokon (@ kokon - Reha für junge Menschen)

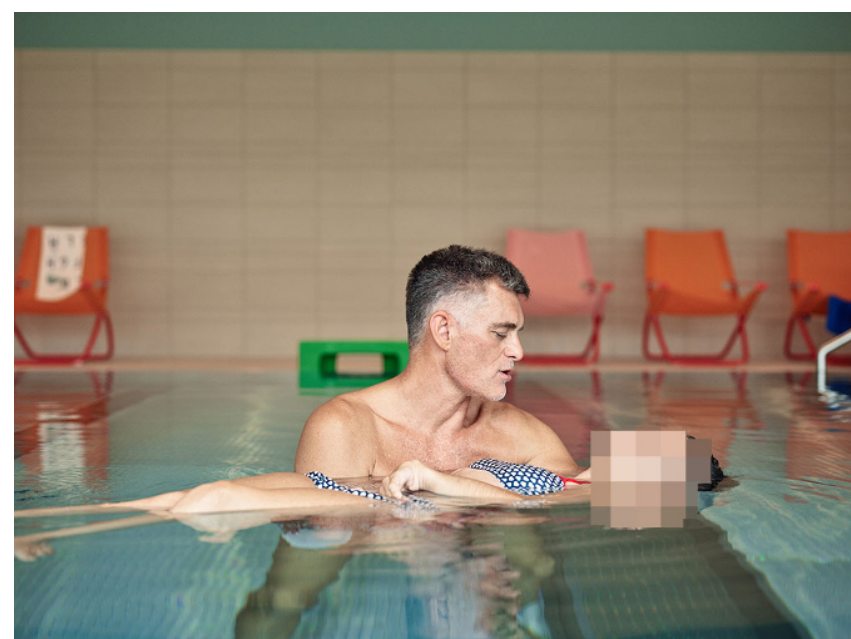

Abb. $4 \Delta$ Wassertherapie im kokon (๔ kokon - Reha für junge Menschen)

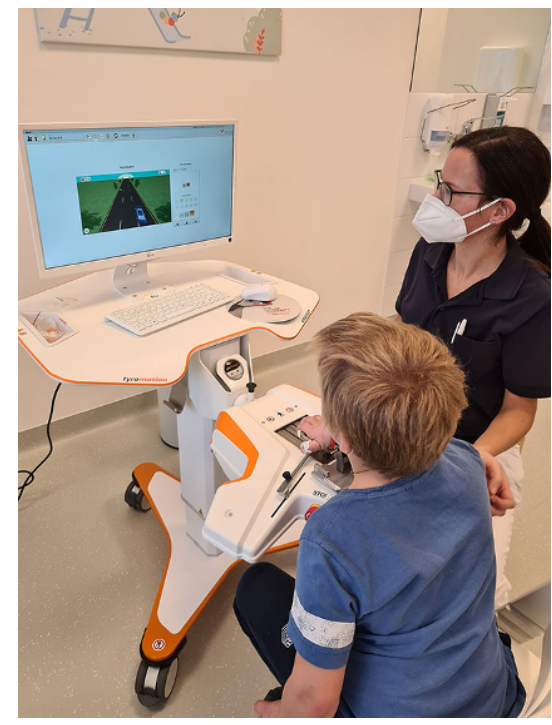

Abb. $5 \Delta$ Amadeo, Tyromotion - computerunterstütztes Training der Handmotorik (@ kokon Reha für junge Menschen)

Health-Schwerpunkt mit mehreren Psychologen und Psychotherapeuten leiten aktuell zwei Fachärzte für Kinder- und Jugendpsychiatrie. Fachärzte für Kinderorthopädie sowie Orthopädietechniker sind regelmäßig im Haus, wir können aber bei Bedarf auch Fachärzte für Kinderchirurgie, Kinderherzchirurgie und Neurochirurgie aus dem Kepler Universitätsklinikum Linz sowie für Kinderurologie aus dem Ordensklinikum Linz Barmherzige Schwestern beiziehen.

\section{Multiprofessionelle Teams mit vielfältiger beruflicher Expertise}

Ein Blick auf die Berufsgruppen im kokon zeigt, wie unterschiedlich diese sind. Sie werden nach Bedarf und Notwendigkeit in das Behandlungskonzept eingebunden.

- Physiotherapie

- Ergotherapie

- Logopädie

- Orthoptik

- Psychologie und Neuropsychologie

- Sportwissenschaften (Leistungsdiagnostik, medizinische Trainingstherapie)

- Diätologie mit Lehrküche

- Heilmassage, Elektrotherapie, Lasertherapie

- Kunst- und Kreativ-, Musik- und Tanztherapie

- Pflege

- Sozialarbeit

- Sozial- und Entwicklungspädagogik

- Kinderbetreuung und Heilstättenschule

Die interdisziplinären Teams erstellen für die Betroffenen optimale Therapieangebote und passen diese während des Aufenthalts laufend an. Dabei setzen wir modernste Technologien wie Galileo-Vibrationsgeräte ein. Computerund robotikunterstützte Reha-Geräte für Bewegungs-, Kraft-, Koordinationsund Gleichgewichtstraining werden $a b$ 2021 das Angebot erweitern (Myro ${ }^{\circledR}$,
Amadeo $^{\circledR}$, Pablo $^{\circledR}$, Tyro $^{\circledR}$, OmegoPlus $^{\circledR}$, Lexo $^{\circledR}$ der Firma Tyromotion).

Auch in der Diagnostik bleibt mit einem Basislabor, Blutgasanalyse, Elektrokardiographie (EKG), Langzeit-EKG, Langzeitblutdruckmessung (LZ-RR), Ultraschall inklusive Echokardiografie, Lungenfunktionsgerät inklusive Belastung und Lyse, Ergometrie und Ergospirometrie fast kein Wunsch offen.

\section{Ganzheitliche Therapie als Erfolgsformel}

Arbeit und Herangehensweise im kokon sind immer individuell. Die Mitarbeiter interessieren sich für das Leben der jungen Menschen, ihre Gewohnheiten und Vorlieben und versuchen mit vielen Tipps und Tricks, deren Alltag bestmöglich zu erleichtern. Dass alle relevanten Experten an einem Ort vereint sind und immer in enger Abstimmung arbeiten, sorgt ebenso für eine erfolgreiche Rehabilitation wie das intensive Zusammensein mit Gleichgesinnten, das die jungen Menschen und ihre Familien als sehr positiv erleben.

Für den ganzheitlichen Ansatz ist auch die Freizeit ein wichtiger Bereich. So können die Rehabilitanden das große Indoor-Schwimmbecken auch über die Zeit der Behandlungen hinaus benutzen und sich auf dem Sportplatz mit Hand-, Fuß-, Volley- oder Basketball austoben. Auch der parkähnliche Garten mit Kinderspieleinrichtungen, Rutschen, Nassspielplatz und Hochbeet ist ideal für Therapie- und für Freizeitaktivitäten. Im 
Gebäude befinden sich eine Turnhalle mit Kletterwand, Tischtennis-, Billard- und Tischfußballtisch, eine Bibliothek, der Kinderspielraum „Abenteuerland“, Jugend- und Aufenthaltsräume mit Großbildfernsehgeräten sowie eine Cafeteria. Auch WLAN gibt es in allen Räumen.

Sozialpädagogen verantworten sämtliche Aktivitäten für Rehabilitanden, sie betreuen unbegleitete Jugendliche, moderieren das Jugendparlament und gestalten verschiedene Veranstaltungen. Normalweise dürfen sich die kleineren Kinder auf Besuche der CliniClowns freuen, die derzeit aber wegen der COVID-19-Schutzmaßnahmen pausieren müssen.

\section{Mobilisierung, kardio- pulmonale Rehabilitation und Mental Health}

Das kokon Rohrbach-Berg umfasst mehrere Indikationen:

1. Mobilisierende Rehabilitation:

- Erkrankungen und Verletzungen des zentralen Nervensystems

- Erkrankungen des peripheren Nervensystems

- Erkrankungen und Verletzungen des Stütz- und Bewegungsapparats

- Folgezustände nach Eingriffen

- Rheumatologie

Als einen aktuellen kokon Kompetenz Schwerpunkt haben wir ein Skoliose-Intensiv-Reha-Konzept für Jugendliche mit juveniler Skoliose entwickelt.

\section{Kardiopulmonale Rehabilitation:}

- Angeborene Fehlbildungen und Erkrankungen des Herz-KreislaufSystems wie Herzfehler vor und nach einer Operation, Fehlbildungssyndrome

- Herzrhythmusstörungen, Kreislaufregulationsstörungen, Kardiomyopathien

- Reha nach einer Herztransplantation und Patienten mit FontanKreislauf

- Erkrankungen der Atemwege

Als kokon Kompetenz Schwerpunkt haben wir aufgrund unserer medizinischen

Paediatr. Paedolog. 2021 · 56:24-29 https://doi.org/10.1007/s00608-020-00860-8

(c) Springer-Verlag GmbH Austria, ein Teil von Springer Nature 2021

\section{J. Emhofer · B. Emhofer $\cdot$ E. Lechner \\ kokon - Rehabilitation für junge Menschen in Rohrbach-Berg}

\section{Zusammenfassung}

kokon Rohrbach-Berg ist das erste österreichische Rehabilitationszentrum, das ausschließlich für Kinder- und Jugendliche von 0-18 Jahren neu errichtet wurde. Im kokon Rohrbach-Berg stehen den Rehabilitanden insgesamt 77 Betten zur Verfügung. Dazu kommen 67 Betten für Begleitpersonen. Das Rehabilitationsangebot umfasst die Indikationsbereiche Mobilisierung, Rehabilitation bei kardiologischen und pulmologischen Erkrankungen sowie die Mental-health-Rehabilitation. Im Mittelpunkt stehen immer die chronisch erkrankten Kinder und Jugendlichen in einer ganzheitlichen
Sichtweise. Das Rehabilitationsprogramm ist komplett auf die Bedürfnisse der Kinder und Jugendlichen ausgerichtet. Die interdisziplinären Teams erstellen individuelle Therapiepläne, helfen mit kindgerechten Angeboten mehr Selbstständigkeit zu erreichen und die Teilhabe am täglichen Leben zu verbessern.

\section{Schlüsselwörter}

Rehabilitation für Kinder und Jugendliche Herz- und Lungenkrankheiten - Neuropädiatrie $\cdot$ Mobilisierung $\cdot$ Kinder- und Jugendpsychiatrie

\section{kokon: Rehabilitation for Young People in Rohrbach-Berg, Austria}

\section{Abstract}

Kokon Rohrbach-Berg is the first Austrian rehabilitation clinic exclusively built for children and teenagers aged $0-18$. There are 77 beds for patients available at kokon Rohrbach-Berg, with an additional 67 beds for accompanying chaperones.

We are specialized in the fields of mobilisation, cardiologic and pulmologic disorders as well as in mental health rehabilitation. Our focus lies on an integrative perception of children and teenagers' chronic illnesses.
The rehabilitation program is designed around the specific needs of each child or teenager.

Our interdisciplinary teams create individual therapy plans, offer child-friendly incentives to further develop independence and strive to improve participation in daily life.

\section{Keywords}

Child and youth rehabilitation . Cardiovascular diseases and pulmonology . Pediatric neurology $\cdot$ Mobilization $\cdot$ Mental health und therapeutischen Expertise die zystische Fibrose gewählt.

\section{Mental-Health-Indikationen:}

- Kinder- und jugendpsychiatrische Erkrankungen sowie entwicklungsund sozialpädiatrische Störungen wie Belastungs- und Anpassungsstörungen

- Depressive Erkrankungen

- Essstörungen in der Stabilisierungsphase

- Angststörungen, Traumafolgestörungen, somatoforme Störungen, Aufmerksamkeitsstörungen, soziale Interaktionsstörungen, Autismus-Spektrum-Störungen, Regulierungsstörungen

- Entwöhnung von Ernährungssonden

\section{Mit homogenen Gruppen Rehabilitationsziele noch besser fördern}

Für alle unsere Indikationen achten wir auf Gemeinsamkeiten der Rehabilitanden und versuchen die Maßnahmen - von der Hygiene bis zu den Behandlungen - gezielt zu steuern:

1. Schon vor der Ankunft achten wir darauf, dass wir möglichst umfassende Informationen von behandelnden Kliniken bzw. Ärzten erhalten. So können wir uns über die Besonderheiten der einzelnen Patienten und über die Erkrankung informieren und den Aufenthalt optimal planen. Das Einholen von Informationen ist wichtig und in der Mental-HealthReha ist eine Kontaktaufnahme mit 


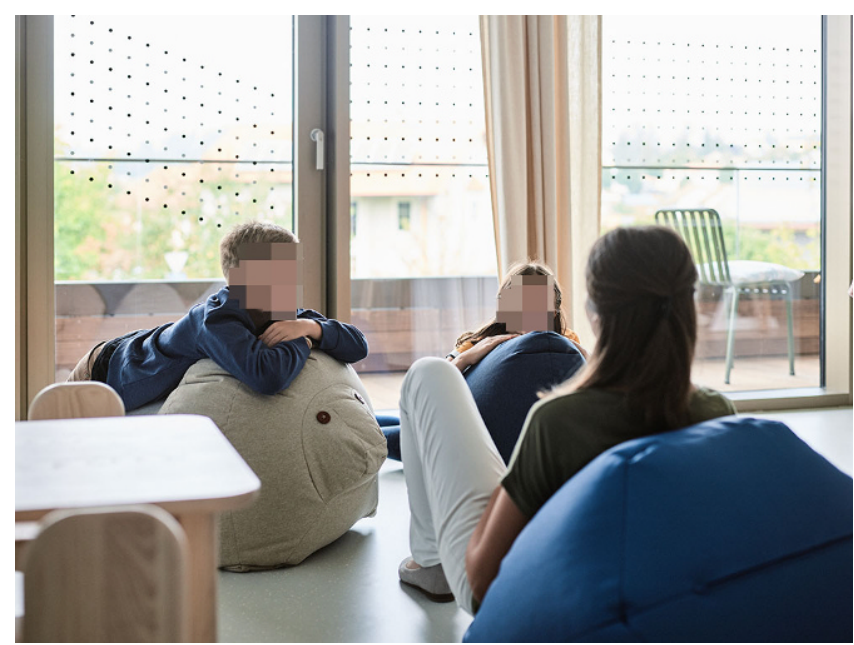

Abb. $6 \Delta$ Jugendliche im Gespräch (@ kokon - Reha für junge Menschen)

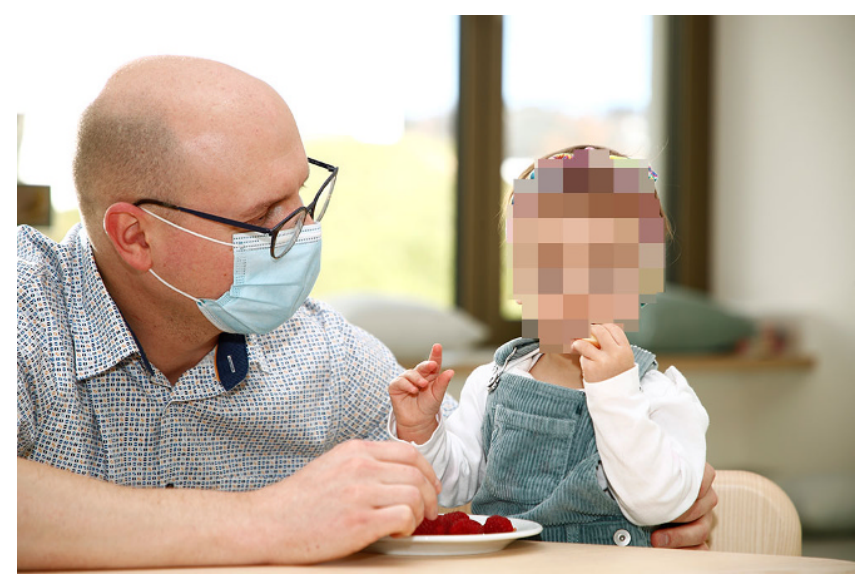

Abb. $8<$ Johanna braucht keine Ernährungssonde mehr, sie hat im kokon essen gelernt (๑ kokon - Reha für junge Menschen)

involvierten Ärzten bzw. Institutionen zur Information sogar zwingend.

2. Bei der Aufnahme führen wir zusätzlich zur ärztlichen Untersuchung - wenn nötig - auch eine Leistungsdiagnostik durch. Skoliose-, HerzKreislauf- und pulmonale Patienten etwa mit zystischer Fibrose brauchen auch eine Ergospirometrie. Bei Herzund Zystische-Fibrose-Erkrankungen müssen wir darüber hinaus auch die medizinische Trainingstherapie gut steuern können.

3. Bei der Belegung sorgen wir dafür, dass sich homogene Gruppen nach Krankheitsbild und Alter bilden können. Durch unsere Skoliose-Turnusse erleben wir deutlich, welche Vorteile Gruppen und ihre Dynamik haben, mitunter ist eine psychologische Begleitung von Vorteil. Schwerpunktmäßig konnten wir für diese Gruppen ein eigenes Programm erarbeiten, das neben Therapiemaßnahmen und Training auch die mentale Schulung und Unterstützung im Sinn einer Krankheitsakzeptanz sowie betreute Gruppenaktivitäten umfasst.

4. Eine absolute Ausnahme von krankheitshomogenen Gruppenbildungen sind Patienten mit zystischer Fibro$s e$. Sie werden nur einzeln und in Kenntnis des aktuellen Keimstatus aufgenommen, danach bestimmen wir die Hygienevorschriften. Für den Umgang von Beschäftigten und Mitpatienten der Betroffenen haben wir ein eigenes Richtlinienpapier erarbeitet. Aktivitäten mit Nichtzystische-Fibrose-Patienten fördern wir, und wenn es das Alter erlaubt, erfassen wir neben regelmäßigen Lungenfunktionsuntersuchungen eine Ergospirometrie.
5. Ein weiterer Schwerpunkt ist die Mental-Health-Rehabilitation. Hier können Kinder auch über das 12. Lebensjahr hinaus begleitet werden. Bei begleiteten Kindern beschäftigen wir uns schwerpunktmäßig mit Fragestellungen rund um kombinierte Entwicklungsrückstände, Regulationsstörungen, allgemeine Ängste, Probleme bei sozialer Anpassung, frühkindlichen Autismus und Aufmerksamkeitsstörungen. Bei unbegleiteten Jugendlichen konzentrieren wir uns auf ADHS, Depressionen nach der Akutbehandlungsphase, posttraumatische Belastungsstörungen und Angststörungen.

\section{Schwerpunkt Mental-Health- Rehabilitation}

Um passende Patientengruppen zu bilden, erfassen wir bei der Mental-HealthRehabilitation Problemstellung und Bedarf vor der Aufnahme besonders intensiv. Dabei richtet sich die Aufnahme in die Gruppen vor allem nach Schwerpunkten der Reha-Ziele, Alter und Entwicklungsstand. Gut durchmischte Gruppen haben den Vorteil, dass in allgemeinen Gruppentherapien eine realitätsnahe Situation wie im Leben außerhalb der Reha geschaffen werden kann. Gemeinsamer Sport, kreative Angebote und Freizeitgestaltung bilden ebenfalls einen realistischen Lebensraum, der später auch in den Alltag nachwirkt. 
Sämtliche Programme schneiden wir auf das Individuum zu und vervollständigen sie durch Einzeltherapien. Um die Situation ganzheitlich $\mathrm{zu}$ erfassen, greifen wir auch familiäre Belastungen, Interaktionsprobleme in Familie, Schule und Kindergarten auf, weil diese fast immer mit den Grunddiagnosen verknüpft sind. Angebote der Elternarbeit erstellen wir aus den Bereichen der Psychologie und Psychotherapie, sie entstehen aber auch in gemischten Elterngruppen, der Eltern-Kind-Musikgruppe und gemeinsamen Spiel- und Bewegungsangeboten. Weil das derzeit noch viel zu wenig in den Konzepten der Kinderrehabilitation berücksichtigt wurde, sehen wir hier einen Nachbesserungsbedarf.

Bei allen Behandlungskonzepten der Mental-Health-Indikationen achten wir auf ein ausgewogenes Maß zwischen Einzel- und Gruppentherapien. Zusätzlich zu den Stärken und sozialen Kompetenzen fördern wir auch motorische, ergotherapeutische und sprachliche Bereiche mit, und in der Heilstättenschule für unsere Pflichtschüler werden ebenfalls Probleme sichtbar und behandelbar gemacht. Für die Konzentrationsförderung und für das kognitive Training setzen wir erprobte computergestützte Programme ein.
Neben den Spezialangeboten bietet kokon auch Gruppen zur Stärkung von Selbstvertrauen, Identitätsfindung, Selbstakzeptanz, Angstbewältigung, Aktivierung und Entspannung an, mit denen Reha-Ziele noch besser erreicht werden können. Einen breiten Raum nehmen neben Sport auch das Trainieren von Alltagsfertigkeiten und das Training zum Erlangen einer altersentsprechenden Autonomie ein. Diese häufig diagnoseübergreifenden Themen können auch in gemischten Gruppen sehr erfolgreich erarbeitet werden. Das Miteinander bei Spiel und Spaß hilft

\section{Hier steht eine Anzeige.}

\section{Springer}


immens dabei, Entwicklungsschritte zu erproben und zu festigen.

\section{Vorbauen für die Zeit nach der Rehabilitation}

Während des drei- bis fünfwöchigen Aufenthalts, der bei Bedarf in zwei kürzere Einheiten aufgeteilt werden kann, leiten wir Maßnahmen zur Nachsorge ein, koordinieren sie und stellen Kontakte zu weiterbetreuenden Gesundheitsdienstleistern und Institutionen her.

Bei schwer chronisch kranken Kindern sollten regelmäßige Rehabilitationsaufenthalte etwa alle ein bis zwei Jahre erfolgen. So können wir die Kinder und Jugendlichen sowie ihre Familien bestmöglich dabei unterstützen, dass ihnen ein aktives, selbstbestimmtes Leben mit höchstmöglicher Teilhabe an den Aktivitäten der jeweiligen Altersgruppe gelingen kann.

\section{Erkenntnisse nach einem Jahr Kinder- und Jugend- rehabilitation}

Nach etwas mehr als einem Jahr Rehabilitationsarbeit mit Kindern, Jugendlichen und ihren Eltern sehen wir bereits folgenden zusätzlichen Bedarf.

\section{Möglichkeit einer Rehabilitation mit Familie}

Die Aufnahme von Begleitpersonen in unserem Haus ist gesichert, allerdings braucht es Förderungen, um das Therapie-Angebot mit Familienorientierung stärker auszuweiten. Dafür bedarf es der Freistellung durch den Arbeitgeber genauso wie der Möglichkeit zur Therapie von Familienmitgliedern (Rehabilitation mit Familie) nicht nur in der onkologischen, sondern auch in der MentalHealth-Reha. Aber ebenso bei anderen Indikationen sollte Reha mit Familie im Bedarfsfall möglich sein, etwa wenn Eltern und Geschwisterkinder von Kleinkindern nach Herztransplantation, mit infauster Prognose, nach schweren Hirnverletzungen oder -erkrankungen einer Therapie und Entlastung bedürfen. Das ist nach dem bisherigen Verrechnungsmodell aber nicht möglich oder kann zumindest nicht verrechnet werden.

\section{Bessere rechtliche Rahmen- bedingungen}

Bereiche wie therapeutische Ausgänge, Wochenendurlaube und Urlaubsfreistellungen sind nach wie vor ungeregelt oder blind an das starre System der Erwachsenenrehabilitation angepasst. Hier braucht es aber besondere Rahmenbedingungen für Kinder und Jugendliche.

Der Ausbau von kokon RohrbachBerg erfolgt seit der Wiederaufnahme nach dem Corona-Lockdown zügig. Die momentane Belegung von etwa 35 bis 38 Rehabilitanden wurde im November auf 50 und wird ab Sommer 2021 auf über 60 gesteigert werden.

\section{Korrespondenzadresse}

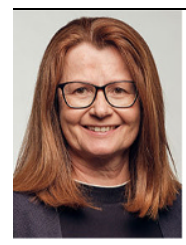

PD Dr. Evelyn Lechner kokon - Reha für junge Menschen

Krankenhausstr. 5,

4150 Rohrbach, Österreich evelyn.lechner@kokon.rehab junge Menschen

\section{Einhaltung ethischer Richtlinien}

Interessenkonflikt. J. Emhofer, B. Emhofer und E. Lechner geben an, dass kein Interessenkonflikt besteht.

Für diesen Beitrag wurden von den Autoren keine Studien an Menschen oder Tieren durchgeführt. Für die aufgeführten Studien gelten die jeweils dort angegebenen ethischen Richtlinien. Für alle Bilder liegen den Autoren die Einverständniserklärungen der Patienten bzw. Eltern vor.

Hinweis des Verlags. Der Verlag bleibt in Hinblick auf geografische Zuordnungen und Gebietsbezeichnungen in veröffentlichten Karten und Institutsadressen neutral. 